\title{
Recuperación y visualización de información en Web of Science y Scopus: una aproximación práctica
}

Cristina Jiménez Noblejas *

Antonio Perianes Rodríguez **

Artículo recibido: 28 de noviembre de 2012. Artículo aceptado: 15 de enero de 2014.

\section{Resumen}

La aparición de Scopus (Elsevier) en noviembre de 2004 supuso un cambio en el mercado internacional de bases de datos y una presión añadida a la supremacía ejercida hasta ese momento por Web of Science (Thomson Reuters). El presente trabajo tiene como propósito la comparación de las posibilidades para recuperar y visualizar información en pantalla a partir del análisis de las interfaces gráficas de usuario y de los principales sistemas de búsqueda de ambas bases de datos. La metodología se basa en el estudio de ambos productos y en la realización de consultas seleccionadas con el exclusivo propósito de conocer el compor-

* Universidad Carlos III de Madrid, España. cristina.jnoblejas@alumnos.uc3m.es

** Universidad Carlos III de Madrid; SCImago Research Group; España.

antonio.perianes@uc3m.es

INVESTIGACIÓN BIBLIOTECOLÓGICA, Vol. 28, Núm.64, septiembre/diciembre, 2014, México, ISSN: 0187-358X. pp. 15-31 
tamiento de Scopus y Web of Science durante el proceso de búsqueda, recuperación y visualización de información. Los resultados obtenidos permiten concluir que si bien las dos bases de datos presentan defectos en la descripción de los registros que afectan su localización, Scopus ofrece mayor eficacia en la obtención de información.

Palabras clave: Web of Science; Scopus; Recuperación de Información; Interfaces Gráficas de Usuario.

\section{Abstract}

Information retrieval and visualization in Web of Science and Scopus: a practical approach

Cristina Jiménez-Noblejas and Antonio Perianes-Rodríguez

The emergence of Scopus (Elsevier) in November 2004 marked a major shift in the international market of databases exerting pressure on the monopoly traditionally held by Web of Science (Thomson Reuters). This paper aims to carry out an analysis of the graphical interfaces of both products as a key factor in the process of information retrieval and visualization. The methodology entails the execution of a series of searches in order to understand the respective performances of Scopus and Web of Science when put through the paces of searching, locating and retrieving information. Although the two databases exhibit deficiencies, results show that Scopus more effectively meets informational requirements in the indexing process by author.

Keywords: Web of Science; Scopus; Information Retrieval; Graphical User Interfaces.

\section{INTRODUCCIÓN}

$\mathrm{D}$ esde la aparición de Science Citation Index (SCI) en los primeros años de la década de los sesenta, germen de Web of Science (en adelante WoS) (Garfield, 1955; 1964), se convirtió en la herramienta de referencia para la satisfacción de las necesidades bibliográficas (citation tracking) de la comuni- 
dad científica. En la actualidad, recoge más de 10000 revistas de investigación y 38 millones registros, y está integrada en la plataforma Web of Knowledge, propiedad de la empresa Thomson Reuters.

En noviembre de 2004 la empresa Elsevier lanzó al mercado la base de datos multidisciplinar Scopus. En sus inicios, Scopus proporcionaba al usuario la posibilidad de consultar el número de veces que un trabajo había sido citado. En la actualidad recoge más de 18000 títulos y 41 millones de registros y se ha convertido en la gran competidora de WoS por el control del mercado internacional de bases de datos de carácter científico.

El surgimiento de Scopus alentó la aparición de una gran cantidad de trabajos con el objeto de identificar cuál de los dos productos era el que mejor respondía a las necesidades de los investigadores. Estos estudios se agruparon en diferentes categorías: aquellos centrados en el análisis individual de Scopus (Fingerman, 2005), aquellos dirigidos a la comparación de las dos bases de datos (Deis \& Goodman, 2005; Fingerman, 2006; LaGuardia, 2005) y aquellos que comparaban ambas con Google Scholar (Jacso, 2005; Bar-Ilan, 2010; Mingers \& Lipitakis, 2010).

En este sentido, Susan Fingerman (2005) realizó un análisis sintético de las principales características de Scopus. En él la autora destacó la cobertura temática de la base de datos, en la que las Ciencias de la Vida y de la Salud copaban el mayor número de títulos. Otro de los aspectos analizados fue su cobertura temporal. El producto de Elsevier permite acceder a los resúmenes de los artículos desde 1966 y al listado completo de referencias bibliográficas desde 1996. Estos datos deben ponerse en relación con WoS, cuya cobertura temporal es significativamente mayor a la de Scopus. Por último, Fingerman señaló el hecho de que el $60 \%$ del contenido recogido en la base de datos no proviene de los Estados Unidos.

Deis \& Goodman (2005) efectuaron un análisis comparativo de ambos productos, dando una severa crítica a Scopus con base en el incumplimiento de la prometida actualización diaria de contenidos y en la falta de algunos artículos, números e incluso años completos de revistas cuya cobertura temporal debía estar cubierta. Este aspecto también ha sido señalado en trabajos posteriores como el de Vieira \& Gomes (2009), en el que se detectó la cobertura parcial de algunos títulos de revistas.

Cheryl LaGuardia (2005) es una firme defensora de Scopus ya que considera que su interfaz está mejor orientada a la búsqueda, utiliza una terminología menos técnica y, por tanto, es más accesible para los usuarios. En relación a WoS reconoce su superioridad en Ciencias Sociales y en Artes y Humanidades. A pesar de ello, recientes estudios como el de Mingers \& Lipitakis (2010) concluyen que la cobertura de WoS es pobre en Ciencias Socia- 
les y, más concretamente, en el área de administración y gestión de empresas, siendo incluso superada por Google Scholar.

En un nuevo estudio comparativo, Fingerman (2006) consideró como una ventaja de Scopus sobre WoS el hecho de incluir tipos documentales como libros, actas de congresos o estudios de mercado, además de los artículos habituales. Posteriormente, la versión 4.10 de WoS incluyó dos nuevas bases de datos referenciales de actas de congresos internacionales: Conference Proceedings Citation Index-Science (CPCI-S) y Conference Proceedings Citation Index-Social Science \& Humanities (CPCI-SSH). Santa \& Herrero-Solana (2010) analizaron la cobertura de las revistas, la producción científica y la visibilidad de los principales países de América Latina y el Caribe en estas dos bases de datos, determinando la mayor cobertura de Scopus en cuanto a revistas, aunque no necesariamente traducida en mayor volumen de trabajos debido a la reciente incorporación de muchos de esos títulos a la base de datos holandesa.

Peter Jacso (2005) fue uno de los primeros autores en realizar un análisis pormenorizado de WoS, Scopus y Google Scholar, prestando especial atención al aumento y a la mejora de las citas así como a los trabajos que de ellas se derivan. Con similares propósitos Judith Bar-Ilan (2010) comparó esas tres mismas fuentes a partir de las citas recibidas por el libro Introduction to informetrics de Leo Egghe y Ronald Rousseau. Una de las principales conclusiones fue que la cobertura de Scopus y WoS es similar si el año de publicación es posterior a 1996.

En esta misma línea se encuentra la principal conclusión del trabajo de Escalona, Lagar \& Pulgarín (2010). Los autores centraron su estudio en el campo de la Ingeniería Química y realizaron un análisis comparativo de ambas bases desde un punto de vista cuantitativo. El resultado de su estudio reflejó la similitud existente entre ellas en lo que a contenido y dinámica se refiere.

Leydesdorff (2012) llevó a cabo una comparación de tendencias de publicación entre Estados Unidos, la Unión Europea (UE-27) y China gracias a una de las últimas novedades de la versión 5.5 de WoS, la cual permite recuperar más de 100000 registros en una única consulta, facilidad ya disponible en Scopus.

A pesar de que han sido numerosos los estudios comparando ambos sistemas de búsqueda, el presente trabajo trata de cubrir, por un lado, el aspecto insuficientemente tratado de la realización de búsquedas específicas para detectar las fortalezas y debilidades de ambos productos; por otro, actualizar resultados previos dado que las novedades en las últimas versiones de ambas bases de datos suponen saltos cualitativos reseñables respecto a las precedentes. 


\section{Metodología}

El principal propósito de este trabajo es establecer la idoneidad de los procesos de obtención de información en Scopus y WoS como herramientas esenciales en los estudios relacionados con la evaluación de la ciencia y la tecnología. Para ello se ha realizado un exhaustivo análisis de ambas interfaces y de los mecanismos de búsqueda que ofrecen, previo a su comparación sistemática, con el fin de establecer las principales fortalezas y debilidades de ambos productos desde la perspectiva de la recuperación y la visualización de los resultados en pantalla.

Para lograr los objetivos planteados se consultaron WoS y Scopus, la primera gracias al contrato suscrito por la Fundación Española para la Ciencia y la Tecnología que la ofrece como servicio público a todas las instituciones públicas de investigación españolas desde enero de 2004 (FECYT, 2009); la segunda a través del servicio de referencia de la Biblioteca Tomás Navarro Tomás del Centro de Ciencias Humanas y Sociales del Consejo Superior de Investigaciones Científicas.

El primer paso consistió en analizar las interfaces de cada una de las bases de datos. A continuación se compararon sus distintas funcionalidades con el propósito de establecer equivalencias y desigualdades desde el punto de vista de la recuperación de información, y finalmente se realizaron consultas en ambas fuentes con idénticos criterios de búsqueda (de autores y palabras en títulos). Para las pesquisas sobre autores se utilizaron las opciones de búsqueda sencilla Search (WoS) y Document Search (Scopus), para las búsquedas de autores se emplearon Author Search (Scopus) y Author Finder (WoS). La búsqueda de palabras en títulos se centró en la localización de términos concretos a través de la búsqueda sencilla (Search de WoS y Document Search de Scopus) y la búsqueda avanzada de WoS y Scopus.

Con ello se pretende, en primer lugar, dar respuesta a cuestiones relacionadas con la calidad de las herramientas gráficas para facilitar el acceso de los usuarios a su materia prima; en segundo lugar, y de forma coyuntural, establecer problemas de descripción que afectan a la localización y visualización de registros en pantalla. En definitiva, se trata de cuestiones que tienden a determinar si estas bases de datos cuentan con instrumentos para resolver los problemas de sinonimia y desambiguación de autores y conceptos, su influencia sobre la descripción de registros o su sensibilidad al uso de idiomas diferentes y signos diacríticos distintivos en las tareas de recuperación de documentos. 


\section{Resultados \\ Interfaces y visualización de resultados}

Ambas bases de datos estructuran su diseño mediante una barra de menús a través de la cual es posible acceder a las principales opciones de búsqueda. Por otra parte, y por lo que al idioma de consulta se refiere, las búsquedas en WoS deben realizarse obligatoriamente en inglés, quedando constancia de ello en las distintas pantallas que constituyen el sitio. Scopus, en cambio, permite el uso de varios idiomas en las consultas.

Las opciones de búsqueda de $W o S$ son las siguientes:

- Search o búsqueda sencilla. La versión más reciente de WoS (5.5) facilita la búsqueda por ResearchID e incluye dos nuevas opciones de refinamiento. Una de ellas es la lematización, que tiene como propósito la extracción del lema o forma consensuada de un término que tiene posibles variantes (plural, masculino o femenino, conjugaciones verbales). La otra opción permite indicar el número de resultados que se visualizarán por página (Figura 1). El campo de búsqueda Author lleva asociado un enlace al asistente de nombre de autor Author Finder.

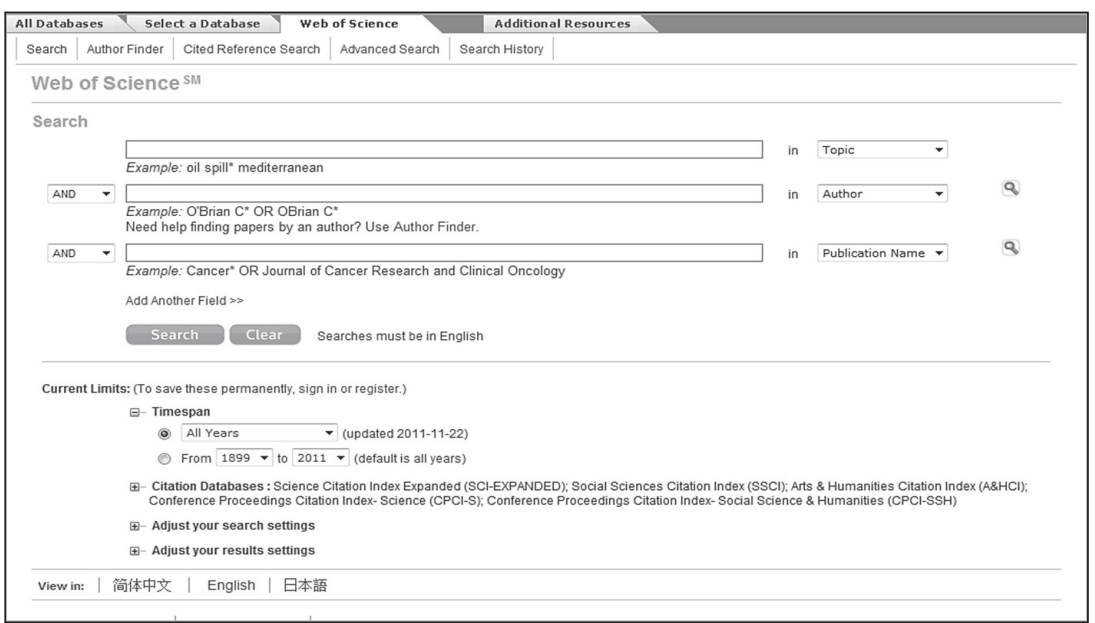

Figura 1. Búsqueda sencilla en WoS (versión 5.5). Fuente: Web of Science.

- Author Finder. En la versión anterior sólo se podía acceder a este asistente desde el campo Author en Search. Actualmente esta opción se incluye en la barra de navegación principal de la base de datos. 
- Cited reference. Localiza artículos que citan un trabajo.

- Advanced Search. Permite hacer consultas complejas mediante el uso de etiquetas de campo, operadores booleanos y paréntesis.

Las principales opciones de búsqueda de Scopus son:

- Document Search. Es la opción de búsqueda sencilla. La pantalla está dividida en dos partes, en la superior se encuentra la zona de consulta y en la inferior se localizan las opciones de refinamiento (Figura 2).

- Author Search. Permite localizar todas las variantes del nombre de un autor registradas en Scopus.

- Affiliation Search. Localiza el nombre y las posibles variantes de una institución.

- Advanced Search. Como en el caso de WoS, es la opción más completa, permitiendo al usuario el uso personalizado de términos, campos y operadores booleanos.

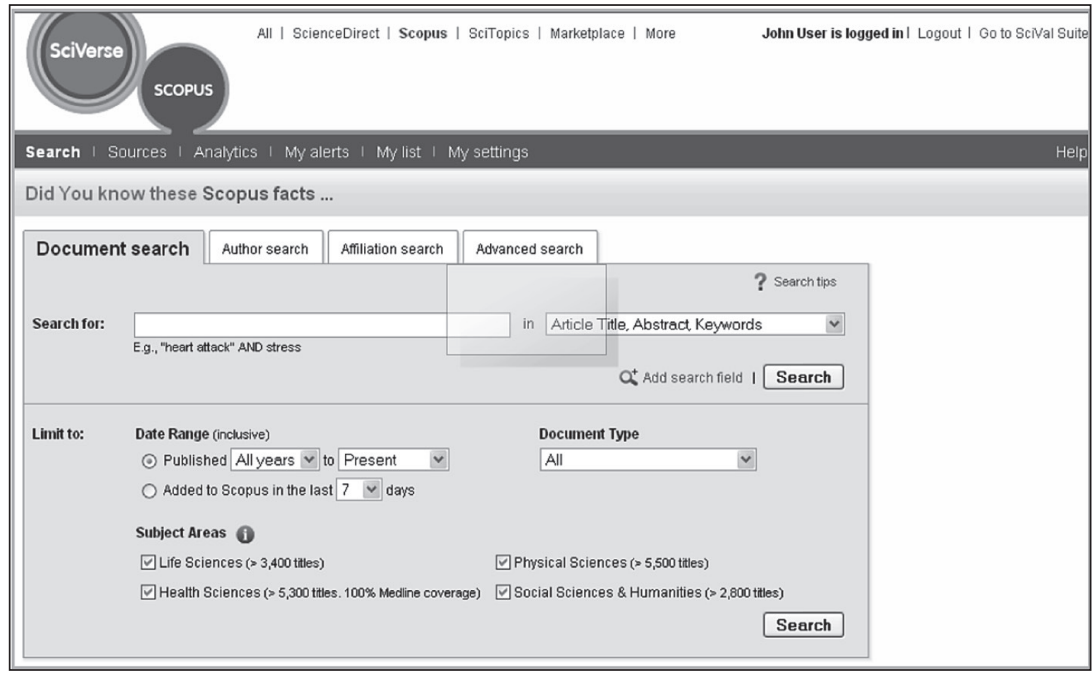

Figura 2. Búsqueda sencilla en Scopus. Fuente: Scopus.

El historial de búsqueda de Scopus (Search history) está presente en la parte inferior de la pantalla de todos los tipos de búsqueda, facilitando así su consulta en todo momento. 


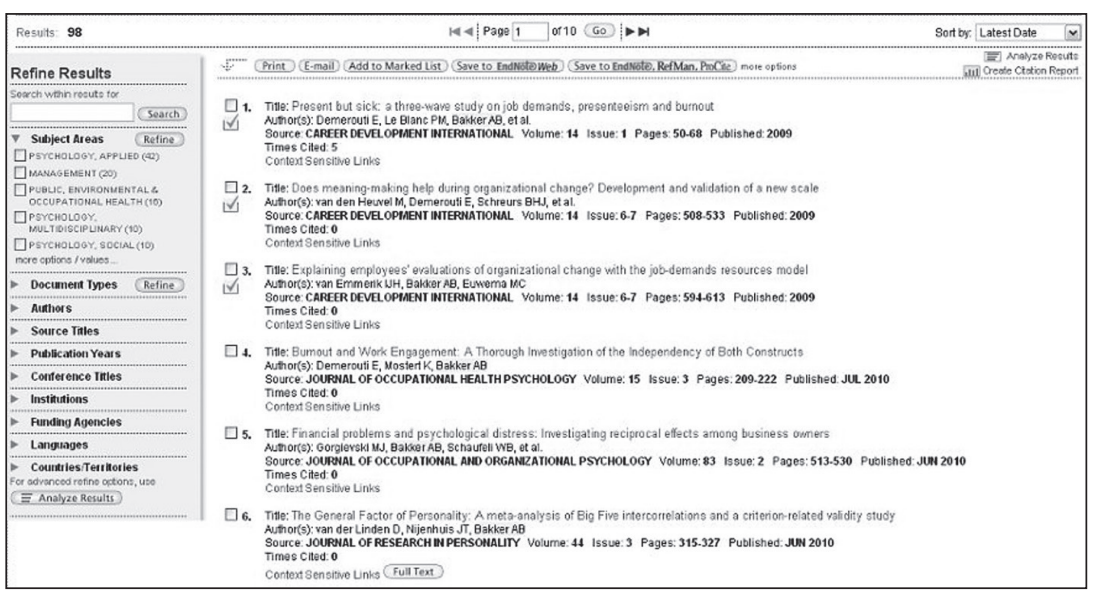

Figura 3. Presentación de resultados en WoS. Fuente: Web of Science.

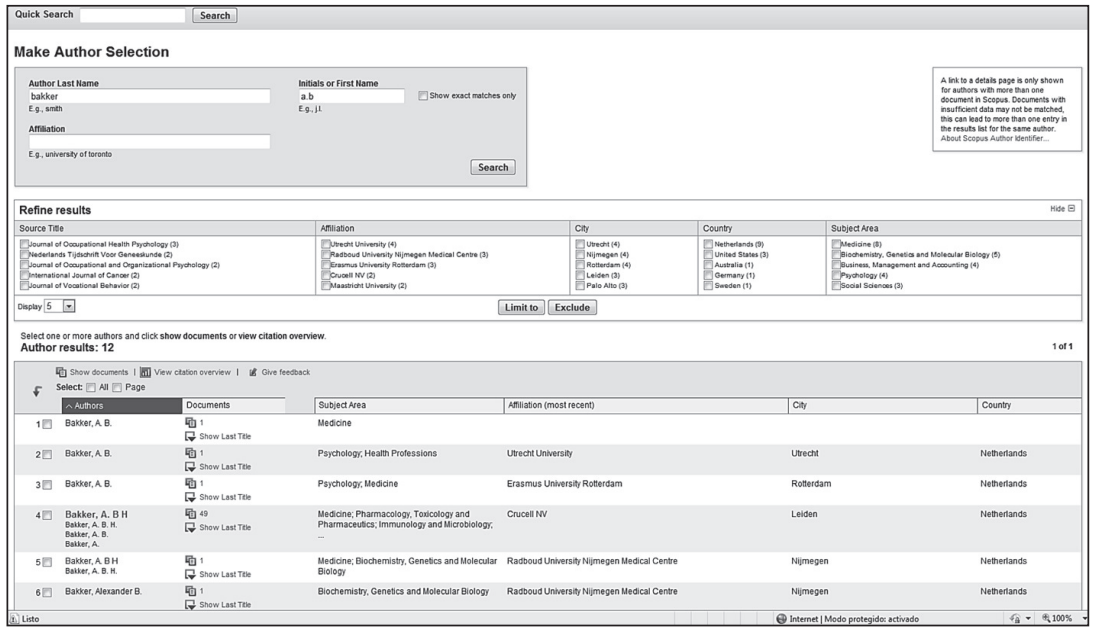

Figura 4. Presentación de resultados en Scopus. Fuente: Scopus.

La página de resultados de ambas bases de datos difiere significativamente en la distribución de la información en la pantalla (Figuras 3 y 4). Mientras que en WoS los resultados se distribuyen en forma de lista, en Scopus se encuentran en la parte inferior, distribuidos en forma tabular, junto con sus funciones asociadas. Por el contrario, las pantallas Full Record (WoS) y Document Details (Scopus) presentan una distribución de contenidos similar en ambas bases de datos. 


\section{Consultas y recuperación de información}

Para probar ambos productos fue necesario el diseño de diversas estrategias de búsqueda que permitieran conocer el grado de adecuación de las interfaces a los usos y necesidades de los usuarios. Estas consultas no son exhaustivas y su única finalidad fue la detección de posibles problemas en la descripción de registros que pudieran afectar de forma determinante la localización y visualización de registros en pantalla. Para ello se plantearon ejemplos de interés para este propósito, con independencia de la temática de las publicaciones, la nacionalidad de los autores o sus filiaciones institucionales.

En la Tabla I se recogen los resultados de las búsquedas de autores realizadas en Search y Author Finder, en el caso de WoS, y Document Search y Author Search de Scopus.

En el caso del primer autor (Arnold B. Bakker) el número de documentos recuperados mediante búsquedas sencillas es similar. En WoS se recuperaron 168 resultados, de los que 44 no le correspondían. En Scopus 35 de los 177 registros no eran del autor.

En el segundo ejemplo (Evangelia Demerouti) el número de documentos recuperados también es parecido. En WoS se recuperaron 55 documentos de los que 7 no eran de la autora. En Scopus se recuperaron 64 documentos, de ellos 4 no eran de la profesora Demerouti.

En el tercer ejemplo (Ana Isabel Sanz Vergel) sí se aprecian variaciones en el número de resultados obtenidos en WoS en función de los términos empleados. Por el contrario, Document Search de Scopus recuperó el mismo número de documentos independientemente de los términos de búsqueda utilizados.

Para el cuarto autor (Daniel Peña) se realizaron consultas utilizando el término "peña" y "pena”. Mientras que Scopus recuperaba documentos con el primero, no ocurría lo mismo en WoS. Esto se debe a que la búsqueda en Scopus permite identificar signos diacríticos, hecho que redunda en la mejora de los resultados de las búsquedas y evitan, por ejemplo, la inclusión de registros de autores apellidados "Pena" en el mismo conjunto de resultados.

En el quinto caso (Mariano Barbacid), tanto en Author Finder como en Author Search el número de resultados es similar, en ambos casos algunos resultados correspondían a la autora María del Mar Barbacid. En el sexto caso (Elizabeth Helen Blackburn), en las dos bases de datos todos los resultados correspondían a la autora cuando se incluían las dos iniciales del nombre.

Finalmente, para los dos últimos autores (François Barré-Sinoussi y José Manuel Sánchez Ron) la opción de búsqueda Document Search (Scopus) recuperó documentos empleando términos que contuvieran tilde, mientras que Search (WoS) no lo hizo. 
Tabla I. Búsquedas comparadas de autores en WoSy Scopus.

\begin{tabular}{|c|c|c|c|c|}
\hline \multirow[t]{2}{*}{ Autores } & WoS & Scopus & WoS & Scopus \\
\hline & Search & Document Search & Author Finder & Author Search \\
\hline Arnold B. Bakker & $\begin{array}{l}\text { bakker a*b* } \\
168 \text { resultados } \\
44 \text { no del autor }\end{array}$ & $\begin{array}{l}\text { bakker, a.b. } \\
177 \text { resultados } \\
35 \text { no del autor }\end{array}$ & $\begin{array}{l}\text { bakker a } \\
398 \text { author sets }\end{array}$ & $\begin{array}{l}\text { bakker a.b. } \\
6 \text { result. (190 doc) } \\
2 \text { result. (147 doc) }\end{array}$ \\
\hline $\begin{array}{l}\text { Evangelia } \\
\text { Demerouti }\end{array}$ & $\begin{array}{l}\text { demerouti } e^{\star} \\
55 \text { resultados } \\
7 \text { no de la autora } \\
\end{array}$ & $\begin{array}{l}\text { demerouti e. } \\
64 \text { resultados } \\
4 \text { no de la autora }\end{array}$ & $\begin{array}{l}\text { demerouti e } \\
17 \text { author sets } \\
10 \text { de la autora }\end{array}$ & $\begin{array}{l}\text { demerouti e. } \\
4 \text { result. (64 docs.) } \\
2 \text { result. (60 docs.) } \\
\end{array}$ \\
\hline $\begin{array}{l}\text { Ana Isabel } \\
\text { Sanz-Vergel }\end{array}$ & $\begin{array}{l}\text { sanz vergel } a^{*} \\
14 \text { resultados } \\
\text { sanz-vergel } a^{*} i^{*} \\
7 \text { resultados } \\
\text { sanz vergel } a^{* i^{*}} \\
7 \text { resultados }\end{array}$ & $\begin{array}{l}\text { sanz vergel, a. } \\
\text { sanz-vergel, a.i. } \\
\text { sanz vergel, a.i. } \\
12 \text { resultados }\end{array}$ & $\begin{array}{l}\text { sanz-vergel, a } \\
7 \text { author sets } \\
\text { sanz-vergel a i } \\
\text { sanz vergel a i } \\
7 \text { author sets todos } \\
\text { de la autora }\end{array}$ & $\begin{array}{l}\text { sanz-vergel a.i. } \\
\text { sanz vergel a.i. } \\
1 \text { result. (12 docs.) }\end{array}$ \\
\hline Daniel Peña & $\begin{array}{l}\text { peña d* } \\
0 \text { resultados } \\
\text { pena d* } \\
532 \text { resultados } \\
69 \text { del autor }\end{array}$ & $\begin{array}{l}\text { peña, d. } \\
973 \text { resultados } \\
\text { Sólo } 60 \text { del autor }\end{array}$ & $\begin{array}{l}\text { peña d } \\
0 \text { author sets } \\
\text { pena d } \\
195 \text { author sets } \\
13 \text { del autor }\end{array}$ & $\begin{array}{l}\text { peña d. } \\
91 \text { result. (1242) } \\
2 \text { result. (78 docs.) }\end{array}$ \\
\hline Mariano Barbacid & \begin{tabular}{|l} 
barbacid $\mathrm{m}^{\star}$ \\
269 resultados \\
9 resultados no del \\
autor \\
barbacid \\
279 resultados \\
11 resultados no \\
del autor \\
\end{tabular} & $\begin{array}{l}\text { barbacid, } m \text {. } \\
\text { barbacid } \\
235 \text { resultados } \\
10 \text { no del autor }\end{array}$ & $\begin{array}{l}\text { barbacid m } \\
35 \text { author sets } \\
31 \text { del autor }\end{array}$ & $\begin{array}{l}\text { barbacid } \mathrm{m} \\
1 \text { result. (232 docs.) }\end{array}$ \\
\hline $\begin{array}{l}\text { Elizabeth Helen } \\
\text { Blackburn }\end{array}$ & $\begin{array}{l}\text { blackburn } e^{\star} \\
432 \text { resultados } \\
332 \text { de la autora } \\
\text { blackburn } e^{*} h^{*} \\
259 \text { resultados to- } \\
\text { dos de la autora }\end{array}$ & $\begin{array}{l}\text { blackburn, e. } \\
507 \text { doc. } \\
227 \text { de autora } \\
\text { blackburn, e.h. } \\
195 \text { todos de la } \\
\text { autora }\end{array}$ & $\begin{array}{l}\text { blackburn eh } \\
481 \text { author sets } \\
\text { 317de la autora }\end{array}$ & $\begin{array}{l}\text { blackburn e.h. } \\
2 \text { result. (225 docs.) }\end{array}$ \\
\hline $\begin{array}{l}\text { Françoise } \\
\text { Barré-Sinoussi }\end{array}$ & $\begin{array}{l}\text { barré-sinoussi f* } \\
0 \text { resultados } \\
\text { barre-sinoussi f* } \\
135 \text { resultados } \\
129 \text { de la autora } \\
\text { barre f* } \\
93 \text { resultados } \\
0 \text { de la autora }\end{array}$ & $\begin{array}{l}\text { barré-sinoussi, } f \\
250 \text { doc. } \\
\text { todos de la autora } \\
\text { barré, } f \\
339 \text { docs. } \\
246 \text { de la autora }\end{array}$ & $\begin{array}{l}\text { barre-sinoussi f } \\
135 \text { author sets } \\
\text { todos de la autora }\end{array}$ & $\begin{array}{l}\text { barré-sinoussif } \\
4 \text { result. (261 docs.) } \\
3 \text { de la autora y uno } \\
\text { dentro de la entra- } \\
\text { da Van Hung, Pham } \\
\text { Pierre }\end{array}$ \\
\hline $\begin{array}{l}\text { José Manuel } \\
\text { Sánchez Ron }\end{array}$ & $\begin{array}{l}\text { sánchez ron j* } m^{\star} \\
0 \text { resultados } \\
\text { sanchez ron j* }{ }^{*}{ }^{*} \\
21 \text { resultados } \\
\text { sanchez-ron j*m } \\
26 \text { resultados } \\
\text { sanchez ron j* }\end{array}$ & $\begin{array}{l}\text { sánchez-ron, j.m. } \\
\text { sánchez ron, j.m. } \\
\text { sánchez-ron,j. } \\
18 \text { docs. } \\
\text { sánchez,j.m. } \\
3634 \text { docs. } \\
\text { sanchez, j. }\end{array}$ & $\begin{array}{l}\text { sanchez-ron j m } \\
106 \text { author sets } \\
26 \text { del autor }\end{array}$ & $\begin{array}{l}\text { sánchez-ron j.m. } \\
1 \text { result. ( } 16 \text { docs.) } \\
\text { todos del autor }\end{array}$ \\
\hline
\end{tabular}




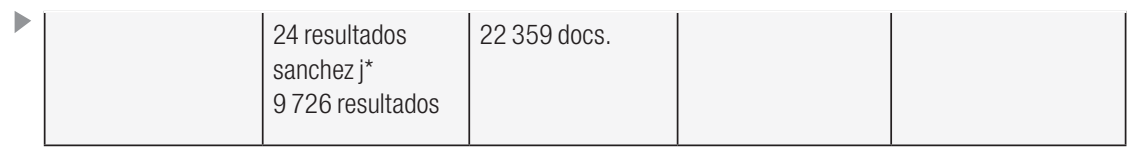

La Tabla II presenta los resultados de las consultas en títulos realizadas a través de las opciones avanzadas en Search (WoS) y Document Search (Scopus). El primer ejemplo tuvo como objeto la recuperación de documentos que contuvieran los términos "biological", "damage" y "animals". Ambas bases de datos recuperaron el mismo documento.

En el segundo ejemplo se trataba de localizar documentos que incluyeran las palabras "recovery" y "burnout" en el título, abstract y palabras clave, y cuyo autor no fuese Arnold B. Bakker. En WoS se obtuvieron 146 documentos, en Scopus 165.

El tercer ejemplo tuvo como propósito recuperar documentos que incluyeran el acrónimo "AIDS" en el título y no incluyeran el acrónimo "HIV". WoS superó los 99000 documentos y Scopus los 54000.

El cuarto ejemplo trató de refinar la consulta anterior, recuperando documentos que contuvieran AIDS y "disease", pero no HIV. El número de resultados se redujo ostensiblemente y la diferencia entre ambas bases de datos también.

El último ejemplo se centró en la recuperación de 8 trabajos de la profesora Sanz Vergel a través de sus títulos. En Scopus se recuperaron 7 documentos, mientras que en $W o S$ sólo fue posible recuperarlos si la consulta incluía los títulos en inglés de los artículos en español.

Tabla Il. Búsquedas comparadas de títulos en WoSy Scopus.

\begin{tabular}{|c|c|c|c|}
\hline Ejemplos & Criterios de búsqueda & WoS & Scopus \\
\hline \multirow[t]{2}{*}{1} & $\begin{array}{l}\text { WoS: } \mathrm{TI}=(\text { biological }) \text { AND TI=(damage) } \text { AND TI=(animals) } \\
\text { Scopus: } \text { TITLE=(BIOLOGICAL) AND TITLE=(DAMAGE) AND TITLE } \\
\text { (ANIMALS) }\end{array}$ & 1 & 1 \\
\hline & $\begin{array}{l}\text { WoS: } \mathrm{TI}=(\text { biological }) \text { AND } T I=(\text { damage }) \text { AND } T I=(\text { animal }) \\
\text { Scopus: } \text { TITLE }=(\text { BIOLOGICAL) AND TITLE }=(\text { DAMAGE) AND TITLE } \\
\text { (ANIMAL) }\end{array}$ & 1 & 1 \\
\hline 2 & $\begin{array}{l}\text { WoS: } \text { TS }=\left(\text { recovery) NOT AU }=\left(\text { BAKKER AB }{ }^{\star}\right) \text { AND TS }=(\text { burnout })\right. \\
\text { Scopus: TITLE-ABS-KEY (RECOVERY) AND TITLE-ABS-KEY (BURNOUT) } \\
\text { AND NOT AUTH (BAKKER AB*) }\end{array}$ & 146 & 165 \\
\hline 3 & $\begin{array}{l}\text { WoS: } \mathrm{TI}=(\mathrm{AIDS}) \text { NOT TI}=(H I V) \\
\text { Scopus: TITLE (AIDS) AND NOT TITLE (HIV) }\end{array}$ & 99160 & 54007 \\
\hline 4 & $\begin{array}{l}\text { WoS: } \text { TI }=\text { (AIDS) AND TI= (disease) NOT TI= (HIV) } \\
\text { Scopus: } \text { TITLE (AIDS) AND TITLE (DISEASE) AND NOT TITLE (HIV) }\end{array}$ & 1897 & 1398 \\
\hline
\end{tabular}




\begin{tabular}{|c|c|c|c|}
\hline \multirow[t]{9}{*}{5} & Topic $=$ & & \\
\hline & $\begin{array}{l}\text { 1. Work-family balance and energy: A day-level study on recovery } \\
\text { conditions }\end{array}$ & 1 & 1 \\
\hline & $\begin{array}{l}\text { 2. Effects of work-family on employees well-being: The moderating role of } \\
\text { recovery strategies? }\end{array}$ & 1 & 1 \\
\hline & $\begin{array}{l}\text { 3. Propiedades psicométricas de la versión española del cuestionario de } \\
\text { interacción trabajo-familia }\end{array}$ & 0 & 1 \\
\hline & $\begin{array}{l}\text { 4. The moderating effects of psychological detachment and thoughts of } \\
\text { revenge in workplace bullying }\end{array}$ & 1 & 1 \\
\hline & $\begin{array}{l}\text { 5. El efecto moderador de la autoestima en el acoso psicológico } \\
\text { en el trabajo }\end{array}$ & 0 & 1 \\
\hline & $\begin{array}{l}\text { 6. El efecto del acoso psicológico en el insomnio: el papel del } \\
\text { distanciamiento psicológico y la rumiación }\end{array}$ & 0 & 1 \\
\hline & $\begin{array}{l}\text { 7. La influencia del estrés de rol y la competencia emocional en el } \\
\text { desgaste profesional y en el engagement }\end{array}$ & 0 & 0 \\
\hline & 8. Estrés traumático secundario: Personalidad y sacudida de creencias & 0 & 1 \\
\hline
\end{tabular}

\section{DisCUSIÓN}

\section{Fortalezas y debilidades de las interfaces de Scopus y WoS}

La interfaz de Scopus mantiene una apariencia uniforme y consistente en todas sus pantallas, presididas por la misma barra de navegación. La última versión de Web of Science analizada presenta, a diferencia de las anteriores, una interfaz mejorada y más integrada, como demuestra la inclusión de las opciones Author Finder y Marked List en la barra principal. Además, muestra de forma más accesible tanto la opción Feedback como el formulario de sugerencias. Sin embargo, el buscador Scientific Web Plus remite a una pantalla cuya apariencia difiere significativamente del resto de la interfaz, afectando la consistencia visual y el uso de las destrezas adquiridas previamente por el usuario.

La página de inicio de Scopus es muy simple, conforme al estilo de los principales buscadores web, con un solo campo de texto por defecto, sin operadores booleanos, con el botón Search fácilmente localizable y la información distribuida de forma sencilla y accesible en la pantalla. Además, el historial de búsqueda de Scopus está al alcance en todo momento, con independencia del tipo de búsqueda empleado, mientras que en WoS es necesario abandonar la página de consultas para poder acceder a las pesquisas realizadas a lo largo de la sesión. 
La forma de realizar las consultas es común en todas las opciones de búsqueda de ambas bases de datos. La última versión de WoS demuestra el gran esfuerzo realizado por Thomson Reuters para que esto sea así, quizá en respuesta a las facilidades de uso que ofrece el producto de su competidor. Muestra de ello es la inclusión de Author Finder como una opción más de búsqueda y no como mero asistente cuyo manejo difería significativamente del requerido por el resto de opciones en versiones anteriores.

\section{Sobre los resultados en búsquedas de autores}

En relación al primer autor, la opción de búsqueda Author Search (Scopus) recuperó 6 sets de documentos de los que sólo 2 pertenecían al autor. El resto de variantes de nombre no se correspondían con el autor buscado:

Bakker, Alexander B H (entrada principal)

Bakker, A. B H

Bakker, A. B.

Bakker, A.

La entrada principal, en negrita, obtuvo 29 documentos de los que ninguno era del autor. Un fenómeno similar se observó en los casos de Barbacid y Demerouti. Por su parte, la opción Author Search de WoS recuperó un conjunto de resultados muy elevado (398), dificultando la recuperación de la obra debido a la excesiva dispersión de los trabajos.

En el caso de la profesora Demerouti, las opciones de refinamiento de resultados de WoS no funcionaron debido a que bajo la entrada DEMEROUTI, E. se encuentran indizadas dos autoras distintas. En este caso, sólo la combinación de autor e institución permitiría lograr la desambiguación de ambas autoras.

En relación al caso de Sanz Vergel, no es posible recuperar todos sus trabajos en Scopus por una descripción incorrecta de la autora (los documentos fueron recogidos bajo la forma Vergel, A.I.S., en la que Sanz se considera como un tercer nombre y no como apellido). En el caso de WoS la incorrecta descripción de la autora hace imposible la recuperación de 7 de sus estudios, de los que hay constancia de localización sin inconvenientes en versiones anteriores de la base de datos.

La consulta en WoS del profesor Peña a partir del término "Pena" recuperó documentos de autores con apellidos "Peña" y "Pena" indistintamente. En el caso de Barbacid, la única entrada a este autor en Author Search (Scopus) es Barbacid, Mariano Mar M., por lo que incluye dentro de este conjunto las obras tanto de Mariano como de María del Mar. 
En relación con la profesora Blackburn, la precisión de los resultados obtenidos varía en función de si se utiliza o no la inicial del segundo nombre. En la búsqueda sencilla los resultados correspondían en su totalidad a la autora si se incluían las dos iniciales del nombre de pila. Por otro lado, el número de sets recuperados a través de WoS era significativamente mayor, 481 en Author Finder, frente a los 2 que ofrecía Author Search (Scopus).

Con respecto a la profesora Barré-Sinoussi la opción de búsqueda Author Finder (WoS) recuperó 135 sets de la autora frente a los 4 de Author Search (Scopus). Sorprende el hecho de que en uno de los resultados de Scopus el trabajo es del autor Hung, Pham Pierre, error que posiblemente provenga de una defectuosa introducción de los datos.

\section{Sobre los resultados en búsquedas en títulos}

En relación al primer ejemplo se ha comprobado que ambas bases de datos utilizan la lematización de términos, que permite la recuperación de documentos utilizando formas alternativas para la búsqueda de un término concreto; por ejemplo, permite recuperar términos en singular y en plural indistintamente.

En el tercer ejemplo se recuperaron documentos con el término "AIDS" referidos a la tercera persona del singular del verbo inglés to aid (ayudar). $\mathrm{Pa}$ ra paliar este problema y reducir el ruido de la tercera consulta, en el cuarto ejemplo se añadió el término "disease" con el fin de recuperar documentos referidos sólo a la enfermedad.

Por último, el ejemplo 5 sirvió para comprobar que son 7 los trabajos de Sanz Vergel incluidos en WoS y Scopus, pero parte de ellos habían sido asignados a la variante de nombre Vergel, A.I.S., dificultando su recuperación y enfatizando los problemas ya evidenciados en las búsquedas de autor de esta profesora.

\section{CONCLusiones}

Las consultas sobre autores han permitido constatar que la principal finalidad del asistente de la opción de búsqueda Author Finder (WoS) es la identificación de las posibles variantes del nombre de un autor, de forma similar a como lo hacía en versión anteriores Scopus con su opción Author Search. Sin embargo, no es infrecuente que los resultados obtenidos agrupen variantes de nombres que corresponden a autores diferentes (como sucede en Scopus con Barbacid o en WoS con Demerouti), hecho que requiere de un esmerado 
trabajo de desambiguación. Por otro lado, en demasiadas ocasiones Author Finder (WoS) obtiene un gran número de author sets, lo que dificulta la recuperación de todas las obras de un determinado autor debido a su excesiva dispersión. En estos casos es recomendable incluir información adicional, como la filiación del autor, para reducir al máximo estos inconvenientes.

Por otra parte, tanto en WoS como en Scopus es importante la inclusión del segundo apellido en las búsquedas de autores latinos o con apellidos compuestos. En las búsquedas de autores anglosajones la inicial del segundo nombre es de vital importancia para evitar ambigüedades indeseadas.

El guión que une los apellidos de los autores es tratado como un espacio en ambas bases de datos y, por tanto, no es un factor que actualmente influya en la recuperación de información aun cuando es necesario para la correcta identificación y adscripción de los trabajos y para evitar que el primer apellido de autores latinos se considere parte del nombre, algo típico en autores anglosajones (el mejor ejemplo es el caso Sanz-Vergel en WoS).

Gracias a las estrategias planteadas ha sido posible comprobar que ambas bases de datos presentan severos problemas de sinonimia y de descripción incorrecta de autores. En este sentido, herramientas como la lematización (recientemente incorporada a WoS en su última versión), que permite recuperar documentos ampliando los términos y conceptos, o iniciativas para la identificación inequívoca de autores como IraLis, Orcid o ResearchID tratan de paliar estos inconvenientes.

La imposibilidad de WoS para incluir tildes, eñes y otros signos diacríticos existentes en idiomas distintos del inglés constituye un inconveniente en la realización de las búsquedas y en la obtención de resultados precisos, además de ser una desventaja frente a Scopus en el mercado internacional no anglosajón. Esta circunstancia es de difícil solución, más allá de incluir información adicional como los datos institucionales en el caso de autores para reducir el exceso de documentos no relevantes.

Han sido numerosas las mejoras que se han producido en la versión 5.5 de WoS relacionadas tanto con el proceso de recuperación de información como con su interfaz gráfica. Sin embargo, son necesarios ajustes adicionales para garantizar la recuperación de las obras de todos los autores, ya que paradójicamente la versión actual impide la recuperación de obras de algunos autores, correctamente descritas y fácilmente recuperables en versiones anteriores.

Elsevier ha creado un producto con una gran interfaz gráfica que, teniendo como referente a Thomson Reuters, ha sabido conservar las virtudes de su predecesor y mejorar sus inconvenientes. WoS, por su parte, ha mejorado la interfaz en su última versión adoptando algunos de los aspectos más destacados de Scopus, como la consistencia visual y la posibilidad de recuperar 
más de 100000 documentos en una única búsqueda. Desde el punto de vista del contenido, Elsevier debe mejorar la cobertura cronológica en general y la temática en áreas como Ciencias Sociales y Artes y Humanidades de Scopus si aspira a competir en igualdad de condiciones con WoS en el mercado de las bases de datos de producción científica y tecnológica.

Finalmente, no debemos olvidar que todos los problemas mencionados, en especial los que atañen a la recuperación de registros adscritos erróneamente por falta de desambiguación o por dificultades para discernir términos con signos diacríticos distintivos, pueden afectar los frecuentes trabajos derivados de estas herramientas, por lo que su solución no sólo redunda en el beneficio de ambas plataformas, sino también en la calidad, robustez y verosimilitud de las docenas de estudios anuales basados en sus datos que afectan a investigadores, instituciones, países y revistas.

\section{BibliografíA}

Bar-Ilan, J. (2010), "Citations to the 'Introduction to infometrics' indexed by WoS, Scopus and Google Scholar", en Scientometrics, 82 (3): 495-506.

Deis, L. \& Goodman, D. (2005), "Web of Science (2004 version) and Scopus", en The Charleston Advisor, 6 (3): 5-21.

Escalona Fernández, M. I.; Lagar Barbosa, P. \& Pulgarín Guerrero, A. (2010), "Web of Science vs. SCOPUS: un estudio cuantitativo en Ingeniería Química", en Anales de Documentación, 13: 159-175.

FECYT (2009), Portal de acceso a la Web of Knowledge, Madrid: Fundación Española para la Ciencia y la Tecnología. Disponible en: http://www.accesowok.fecyt.es/?page_id=2329 [Fecha de consulta: 06/11/2012].

Fingerman, S. (2005), "SCOPUS: Profusion and confusion", en Online, $29(2): 36-38$.

- (2006), "Web of Science and Scopus: current features and capabilities", en Issues in Science and Technology Librarianship, 48. Disponible en: http://www.istl.org/06-fall/electronic2.html [Fecha de consulta: 06/11/2012].

Garfield, E. (1955), "Citation indexes for science: a new dimension in documentation through association of ideas", en Science, 122 (3159): $108-111$.

(1964), "Science Citation Index: a new dimension in indexing", en Science, 144 (361): 649-654.

Jacso, P. (2005), "As we may search-Comparison of major features of Web of Science, Scopus, and Google Scholar citation-based and citation-enhanced databases", en Current Science, 89 (9): 1537-1547. 
LaGuardia, C. (2005), "E-Views and Reviews: Scopus vs. Web of Science", en Library Journal, January. Disponible en: http://www.libraryjournal.com/article/CA491154.html [Fecha de consulta: 06/ 11/2012].

Leydesdorff, L. (2012), "World shares of publications of the USA, EU27, and China compared and predicted using the new Web of $S_{C i}$ ence interface versus Scopus", en El Profesional de la Información, 21: 43-49.

Mingers, J. \& Lipitakis, E. (2010), "Counting the citations; a comparison of Web of Science and Google Scholar in the field of business and management", en Scientometrics, 85 (2): 613-625.

Santa, S. \& Herrero-Solana, V. (2010), "Cobertura de la ciencia de América Latina y el Caribe en Scopus vs. Web of Science”, en Investigación Bibliotecológica, 24 (52): 13-27.

Scopus (2010), User guide, Amsterdam: Elsevier.

Vieira, E. S. \& Gomes, J. (2009), "A comparison of Scopus and Web of Science for a typical university”, en Scientometrics, 81 (2): 587-600.

$\infty$ 